\title{
Fluid intake patterns of adults: results of six Liq.In7 national cross-sectional surveys
}

\author{
Isabelle Guelinckx ${ }^{1}$, Clémentine Morin ${ }^{1}$, Luis A. Moreno ${ }^{2,3}$, Stavros A. Kavouras ${ }^{4}$, \\ Jordi Salas-Salvadó ${ }^{5,3}$, Homero Martinez ${ }^{6}$ and Joan Gandy ${ }^{7,8}$ \\ ${ }^{1}$ Department of Hydration \& Health, Danone Research, Palaiseau, France, \\ ${ }^{2}$ GENUD (Growth, Exercise, Nutrition and Development) Research Group, Faculty of Health Sciences, Universidad de \\ Zaragoza, Instituto Agroalimentario de Aragón (IA2), Instituto Investigación Sanitaria Aragón (IIS Aragón), \\ Zaragoza, Spain, \\ ${ }^{3}$ CIBERobn (Centro de Investigación Biomédica en Red Fisiopatología de la Obesidad y Nutrición), Institute of Health \\ Carlos III, Madrid, Spain, \\ ${ }^{4}$ Hydration Science Lab, Arizona State University, Phoenix, AZ, USA, \\ ${ }^{5}$ Human Nutrition Unit, Hospital Universitari de Sant Joan de Reus, Faculty of Medicine and Health Sciences, Institut \\ d'Investigació Sanitària Pere Virgili, Biochemistry and Biotechnology Department, Universitat Rovira i Virgili, Reus, \\ Spain, \\ ${ }^{6}$ Hospital Infantil de México Federico Gómez, Mexico City, Mexico, \\ ${ }^{7}$ British Dietetic Association, Birmingham, United Kingdom. and \\ ${ }^{8}$ School of Life and Medical Sciences, University of Hertfordshire, Hatfield, United Kingdom
}

\section{Abstract}

Dietary patterns provide valuable information for the development of public health policies. Despite increasing interest in the role of fluids in health and disease there are limited data on drinking patterns in adults. The aim of this analysis was to identify and characterize patterns of fluid intake in adults in cross-sectional surveys in China, Indonesia, Argentina, Brazil, Mexico and Uruguay.

Data on fluid intake volume and type amongst 8159 adults $(46 \%$ male) were collected using a validated 7 -day fluid specific record (Liq. $\left.\mathrm{In}^{7}\right)$. A cluster analysis (partitioning around k-medoids algorithm) was used to identify relatively distinct clusters of subjects based on eight fluid types (water, milk and its derivatives, hot beverages (HB), sugar sweetened beverages (SSB), 100\% fruit juices, artificial/non-nutritive sweetened beverages, alcoholic beverages, other beverages). Subjects were categorized according to total daily fluid intake as low $(<1500 \mathrm{~mL} / \mathrm{d})$, medium $(>1500-<3000 \mathrm{~mL} / \mathrm{d})$, high $(>3000 \mathrm{~mL}-<4000 \mathrm{~mL} / \mathrm{d})$ or very high $(>4000 \mathrm{~mL} / \mathrm{d})$. The clusters were characterized according to country of residence, socio-demographics and lifestyle factors.

The eight interpretable clusters identified were: (1) low drinkers - SSB ( $n=2450)$, (2) low drinkers - HB ( $n=1589)$, (3) medium drinkers - water $(n=1306)$, (4) medium drinkers - HB and SSB $(n=892)$, (5) medium drinkers - SSB $(n=262)$, (6) high drinkers - water $(n=817),(7)$ high drinkers - water \& SSB $(n=313)$ and $(8)$ very high drinkers - water $(n=530)$. Country of residence was the dominant characteristic in all clusters. In cluster \#1 34\% of subjects were resident in Mexico (v test 21 ), $43 \%$ of cluster \#2 lived in China (v test 21.4). 47\% of cluster \#4 lived in Argentina (v test 24.2) with 30\% living in Uruguay (v test 19.7), (v test 8). Cluster \#3 was characterized by residence in Indonesia (v test 23.6) as were clusters \#6 (v test 28.5), cluster \#7 (v test 8.2) and cluster \#8 (v test 24.5). To a lesser extent cluster \#7 (high drinkers - water \& SSB) was characterised by residence in Mexico (v test 3.6). In cluster \#5, 51\% and 30\% lived in Mexico and Argentina, respectively; with $60 \%$ of this cluster being male.

Fluid intake patterns (clusters) varied according to the different countries. This information highlights the need to tailor interventions aimed at improving fluid intake behaviour and health to the specific adult populations.

\section{Conflict of Interest}

CM and IG are full time employee of Danone Research. JS-S, LAM, SAK, JG, HM are members of the advisory board on fluid intake of Danone Research, and have received consultancies from Danone Research. SAK had active research grants from Danone Research and he is a consultant for Standard Process. JS-S and LAM has received consultancies from Danone S.A. 